\author{
С. С. Гайдук ${ }^{1}$ В. М. Чудук ${ }^{2}$ \\ ${ }^{1}$ Белорусский государственный технологический университет \\ ${ }^{2}$ ЧПТУП «Линалис плюс»
}

\title{
АВТОМАТИЗАЦИЯ ПРОЕКТИРОВАНИЯ ИЗДЕЛИЙ ИЗ ДРЕВЕСИНЫ
}

\begin{abstract}
Проведенная работа посвящена исследованию систем автоматизированного проектирования изделий из древесины, а также разработке и теоретическому обоснованию проектирования сложной мебели и столярных изделий. В рамках проведенной работы было использовано два метода проектирования сложных изделий. Первый подход «деталь-сборка» подразумевал сборку в единый узел смоделированных и сохраненных ранее деталей: сначала размещенных в пространстве, сопряженных вместе и зафиксированных. Это способ моделирования привел к насыщению проекта похожими деталями разной конфигурации, что потребовало увеличения временных затрат и мощности компьютера на обработку связей между деталями. Второй способ основывался на многотельной детали, состоящей из нескольких твердых тел. В этом случае отдельные детали изделия создавались в одном документе многотельной детали, что способствовало сокращению сложных связей между элементами. Для созданных моделей автокомпонентов мебельной фурнитуры разработана схема автоматизированного размещения основного параметрического элемента, давшая возможность в автоматическом режиме изменять размеры в зависимости от габаритов соединяемых деталей. Использование предложенной методики на предприятии позволило спроектировать трехмерную модель изделия, на основании которой был получен полный комплекс конструкторской документации в соответствии с требованиями действующих стандартов, и снизить время на проектирование до 20\% по сравнению с классическими методами проектирования.
\end{abstract}

Ключевые слова: изделие, древесина, автоматизация, проектирование, деталь, сборка, многотельная модель.

Для цитирования: Гайдук С. С., Чудук В. М. Автоматизация проектирования изделий из древесины // Труды БГТУ. Сер. 1, Лесное хоз-во, природопользование и перераб. возобновляемых ресурсов. 2022. № 1 (252). С. 181-187.

\author{
S. S. Haiduk ${ }^{1}$, V. M. Chuduk ${ }^{2}$ \\ ${ }^{1}$ Belarusian State Technological University \\ ${ }^{2}$ PPTUE "Linalis plus"
}

\section{AUTOMATION OF DESIGNING WOOD PRODUCTS}

The work is devoted to the study of computer-aided design of wood products, as well as the development and theoretical justification of the design of complex furniture and joinery. As part of the work carried out, two methods of designing complex products were used. The first "partassembly" approach meant the assembly into a single node of previously modeled and stored parts: first placed in space, paired together and fixed. This method of modeling led to the saturation of the project with similar parts of different configurations, which required an increase in the time costs and computer power to process the connections between the parts. The second method was based on a multibody part consisting of several solids. In this case, individual parts of the product were created in one document of a multi-body part, which contributed to the reduction of complex connections between elements. For the created models of auto-components of furniture fittings, a scheme of automated placement of the main parametric element was developed, which made it possible to automatically change the dimensions depending on the dimensions of the connected parts. The use of the proposed methodology at the enterprise made it possible to design a three-dimensional model of the product, on the basis of which a full set of design documentation was obtained in accordance with the requirements of current standards, and to reduce the design time by up to $20 \%$ compared with classical design methods.

Key words: product, wood, automation, design, detail, assembling, multibody model.

For citation: Haiduk S. S., Chuduk V. M. Automation of designing wood products. Proceedings of BSTU, issue 1, Forestry. Nature Management. Processing of Renewable Resources, 2022, no. 1 (252), pp. 181-187 (In Russian). 
Введение. Республика Беларусь по ряду ключевых показателей, характеризующих лесной фонд (лесистость территории, площадь лесов и запас растущей древесины в пересчете на одного жителя), входит в первую десятку лесных государств Европы. По данным Министерства лесного хозяйства, лесистость республики составляет $40,1 \%$, общий запас древостоя - 1831,8 млн м ${ }^{3}$, а доля спелых и перестойных лесов - 16,8\% [1]. Наличие широкой сырьевой базы способствует развитию деревообрабатывающей промышленности, объем которой во внутреннем валовом продукте составляет порядка 4\%. Наибольший вклад вносит мебельная промышленность, годовой объем производства которой в 2020 г. достиг 2,15 млрд бел. руб. [2].

Увеличение объемов выпуска продукции и повышение ее конкурентоспособности требует постоянного внедрения инноваций. Одним из перспективнейших направлений научно-технического прогресса в этой области можно считать разработку, внедрение и совершенствование систем автоматизированного проектирования (САПР). Это объясняется тем, что промышленный потенциал определяется не только возможностями массового производства новейших изделий техники, но и возможностями их быстрого проектирования. Так как количество вновь разрабатываемых отраслями промышленности изделий удваивается каждые 15 лет, а их сложность каждые 10 лет, то требования к сроку и качеству их проектирования непрерывно растут. В мире производительность труда в промышленности за последние 100 лет в среднем возросла на $100 \%$, а рост производительности труда в проектировании только на 20\%. Поэтому внедрение средств вычислительной техники в практику проектирования на системной основе, создание систем автоматизированного проектирования позволит устранить это противоречие, поскольку САПР является своеобразным конвейером для проектирования соответствующих изделий.

Срок исполнения заказов на мебельные изделия зачастую так же важен, как и их приемлемая цена или качество. По этой причине все операции - от разработки дизайна до отгрузки готовой продукции - должны выполняться быстро, без лишних затрат на передачу информации или ее доработку.

В настоящее время автоматизация стала неотъемлемой частью производственных процессов на деревообрабатывающих и мебельных предприятиях. Наиболее распространенной практикой является использование частичной автоматизации для внедрения на тех участках, где образуются так называемые «узкие» места. Следует отметить, что частичная автоматизация, безусловно, дает новый импульс развитию предприятия, но она не в полной мере способна обеспечить общий глобальный успех [3-6].

Целью проводимых исследований являлась оценка САПР для проектирования изделий из древесины, а также первый этап комплексной автоматизации предприятия, а именно разработка и теоретическое обоснование проектирования сложной мебели и столярных изделий.

Основная часть. САПР - это организационнотехническая система, в рамках которой группа проектировщиков при помощи специализированного программно-аппаратного комплекса создает математические модели изделий и рассчитывает их, формирует необходимую документацию и осуществляет экспертные оценки принимаемых решений.

Программы для проектирования изделий из древесины разделяют на две категории: дизайнерские и конструкторские. С помощью дизайнерских можно расставлять готовые мебельные модули в помещении, подсчитывать сумму заказа, быстро визуализировать интерьер помещения, предоставлять графическую информацию клиентам.

Конструкторские программы дают намного больше возможностей. С их помощью можно разрабатывать новые модели мебели, новые прототипы, они обеспечивают большую точность чертежей, позволяют вести учет материалов. Кроме того, дают возможность генерировать файлы для станков с ЧПУ, делать карты раскроя и многое другое.

Все эти программы также можно разделить еще на следующие типы. Базовые программы интерактивной графики. К ним можно отнести комплексы программ на основе AutoCAD, Копас-3D, T-FLEX CAD, Solidworks. Специализированные программы, к которым относят: bCAD, K3 Meбель, WOOD, CADWork и др. Офисные программы типа Pro100 и Visio [5-8].

Программа Базис-Мебельщик основана на математическом ядре $\mathrm{C} 3 \mathrm{D}$ компанией $\mathrm{ACKOH} \mathrm{и}$ считается лидером мебельного софта. Она состоит из нескольких модулей. К плюсам программы относят: отличный графический редактор, с помощью которого можно получать профессиональные чертежи и схемы для раскроя, изготовления деталей и сборки; возможность создания собственных библиотек; функция автоматической привязки маркера; удобная расстановка крепежа с возможностью создания аналоговых схем. Самым большим недостатком является высокая цена программного обеспечения, а также недостаточно высокая визуализация и сложность при освоении $[9,10]$.

Высокая степень визуализации представлена в программе PRO100. Эта программа является достаточно простой в освоении, в ней есть функция 
расчета предполагаемой стоимости будущей мебели. Однако она не имеет набора «привязок», с помощью которых можно более точно позиционировать деталь, нет и возможности создать карту присадок [11].

Программа T-FLEX создана на геометрическом ядре Parasolid [12]. Программа позволяет автоматизировать все этапы работы с изделием - от оформления заказа до проектирования и изготовления мебели, учитывая особенности как серийных, так и единичных типов производств. Система имеет модульную структуру и разделена на редакции. Каждая редакция представляет собой отдельную программу, которая может работать как в составе единого комплекса, так и автономно. Параметризация системы представляет собой неограниченные возможности для разработки серийных и заказных прототипов мебели, а богатый функционал 2D-черчения позволяет создавать чертежи и эскизы в соответствии с ЕСКД $[7,11]$.

Программа Solidworks по своим свойствам очень схожа с T-FLEX. Комплексные решения SolidWorks базируются на передовых технологиях гибридного параметрического моделирования и широком спектре специализированных модулей. Программное обеспечение функционирует на платформе Windows, поддерживает ГОСТ и ЕСКД [13].

3D Studio MAX построена на графическом ядре Nitrous. Программа трехмерного моделирования и анимации 3D Studio MAX позволяет создать компьютерный дизайн интерьера, смоделировать стены, двери, мебель, текстуру объекта. С помощью ее можно создавать фотореалистичные изображения изделий и интерьеров, однако она не дает ни чертежей, ни карт раскроя, ни стоимости проекта. Также создание проекта не является быстрым процессом.

В качестве примера использования рассмотрим программный продукт SolidWorks, позволяющий создавать трехмерные модели будущих изделий, с помощью которых можно оценить конструкцию и провести исследования различных свойств проектируемого изделия.

Готовые мебельные и столярные изделия включают в себя множество деталей, подсборок и сборок. Наличие большого количества компонентов изделия вызывает необходимость выбора способа их оптимальной и логической организации в проекте. Часто встречаются такие узлы, как рамочно-филенчатые фасады, мебельные ящики, цокольные коробки и некоторые корпуса, которые являются подсборками изделия, зачастую однотипными, но с различными конфигурациями и типоразмерами. В процессе проектирования таких узлов конструктор сталкивается с двумя принципиальными подходами [14-16]:
- проектирование в среде «деталь-сборка»;

- проектирование в среде многотельной детали.

В зависимости от того, какие поставленные вопросы и задачи нужно решить конструктору, происходит выбор среды проектирования. Моделирование техникой «деталь-сборка» применимо в случаях:

- промышленного дизайна;

- расширенных требований для операций на оборудовании с ЧПУ;

- как метод для стандартных конструкционных решений;

- проектирования серийной мебели;

- как метод проектирования для большого штата сотрудников;

- как метод получения информации о материалах и спецификациях.

Моделирование техникой многотельной детали применимо в случаях:

- концептуального и промышленного дизайна;

- как средство связи вида и формы изделия;

- быстрого моделирования;

- как метод для проектирования индивидуальной мебели на заказ;

- как метод получения информации о материалах и спецификациях.

Первый способ является классическим. Трехмерная деталь является основным стандартным блоком программного обеспечения механического проектирования SolidWorks. В документе «Сборка» собираются в единый узел смоделированные и сохраненные ранее детали: сначала размещаются в пространстве, сопрягаются вместе и фиксируются (рис. 1).

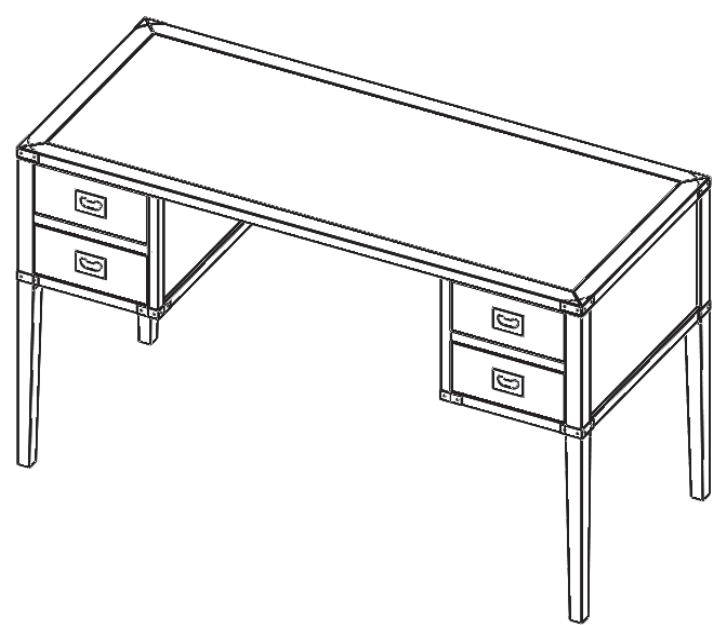

Рис. 1. Стол письменный, спроектированный в среде «деталь-сборка»

Моделирование подсборок изделий данным способом ведет к насыщению проекта похожими деталями разной конфигурации: брусками, филенками, штапиками, раскладками, карнизами и т. д. Вследствие этого увеличивается потребность в 
вычислительных мощностях компьютера, а также временных затратах на организацию и преобразование подсборок и включение их в конечную сборку. Также от конструктора требуется постоянная концентрация при многократном копировании таких узлов. Копирование подсборки ведет к автоматическому копированию входящих в нее деталей, а если детали ссылались на исходную сборку (были спроектированы в контексте сборки с использованием внешних ссылок), нарушение алгоритма копирования приведет к разрушению сопряжений в подсборке.

Второй способ проектирования узлов мебели основывается на многотельных деталях. Многотельная деталь состоит из нескольких твердых тел, которые не являются динамическими (рис. 2). Однако, если необходимо представить динамическое перемещение тел, следует использовать сборку.

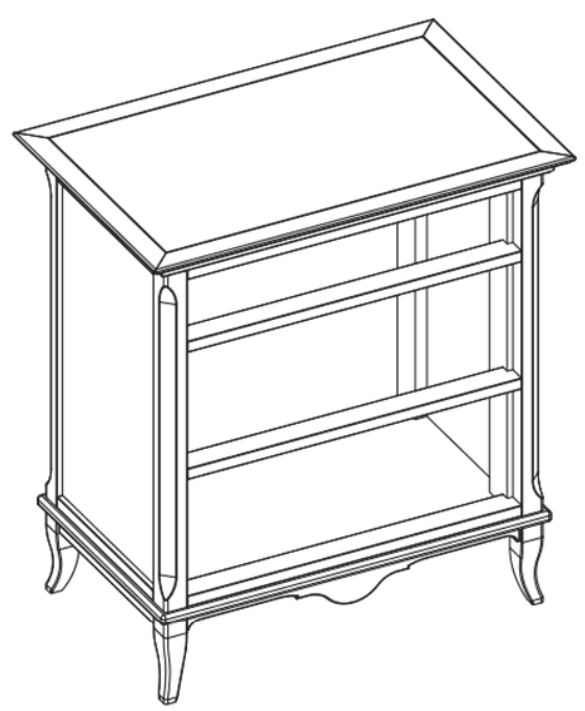

Рис. 2. Каркас комода, спроектированного в среде многотельной детали

При таком подходе не требуется создавать каждую деталь узла по отдельности и в отдельном файле. Простыми операциями выталкивания создаются отдельные панели корпуса (стенки, полики, перегородкии и т. д.), операцией «бобышка по траектории» проектируются целые комплекты штапиков, рамок, карнизов в одном документе многотельной детали. В таком документе не существует сопряжений, которые могут быть разрушены.

При активации опции «сварная деталь» конструктор может создавать «списки вырезов», которые являются аналогами спецификация для сборок. В дереве конструирования все твердые тела автоматически сортируются по папкам в соответствии с их геометрическим подобием.

Для каждой такой группы тел есть возможность создать граничную рамку, которая представляет собой трехмерный эскиз, и ее основание по умолчанию находится на плоскости X-Y. Исходя из ориентации граничной рамки, она является наименьшей рамкой, охватывающей тело. Таким образом, для многотельной детали конструктор автоматически получает спецификацию элементов с их габаритными размерами, без использования формул, свойств, ссылок и т. д.

При работе с многотельной деталью материал можно применить ко всем телам детали сразу либо к одному или нескольким отдельным телам. Поэтому для исследования нет разницы, будет ли это сборка или многотельная деталь, так же как и для чертежа.

Многотельный узел по определению является параметрическим. Например, изменение габаритного размера фасада ведет к перестроению филенки и брусков (рис. 3).
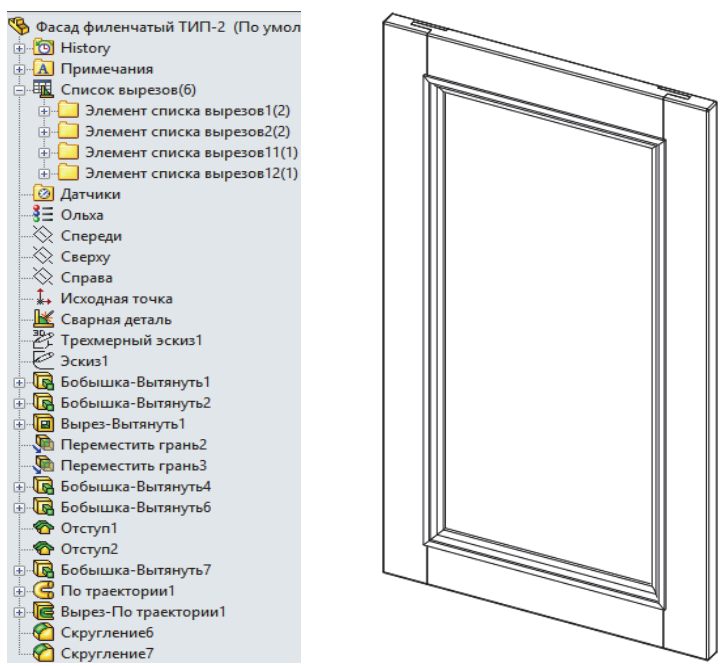

Рис. 3. Автоматический «список вырезов» в многотельной детали на примере параметрического фасада

Конструктор не затрачивает время на создание формул, параметров и алгоритмов для этого процесса. Копирование таких узлов можно осуществлять в проводнике без риска разрушения каких-либо ссылок. Копировать нужно только один файл, а не группу файлов, как в случае со сборкой.

Можно сохранять сборку как документ многотельной детали. Это позволяет сохранять сложные сборки как документы деталей меньшего размера, что обеспечит более удобное совместное использование файлов. Например, имеется проект сложной кухни, и потенциальному клиенту требуется расположить ее в планировке помещения для визуализации. Можно сохранить сборку кухни как документ детали и отправить файл детали потенциальному клиенту без риска нарушить целостность проекта и без необходимости передавать файл документа сборки большого размера. 
Для повышения производительности проектирования в САПР используются библиотеки, шаблоны и автокомпоненты. Разработка правильных шаблонов и форматов гарантирует возможность сэкономить время оформления чертежей путем автоматизации многих общих задач с использованием шаблонов, нескольких форматов, блоков, избранных и связанных пользовательских свойств.

Автокомпоненты - это детали или узлы, которые можно разместить на верхнем уровне сборки вместе с требуемыми монтажными действиями и элементами (вырезы, пазы, отверстия, крепежные элементы и т. д.). Автокомпоненты могут настраиваться, могут автоматически изменять свои размеры, а также располагаться на цилиндрических поверхностях. Так, в качестве автокомпонента может выступать мебельная крепежная или лицевая фурнитура (рис. 4), которая создана в среде многотельной детали.

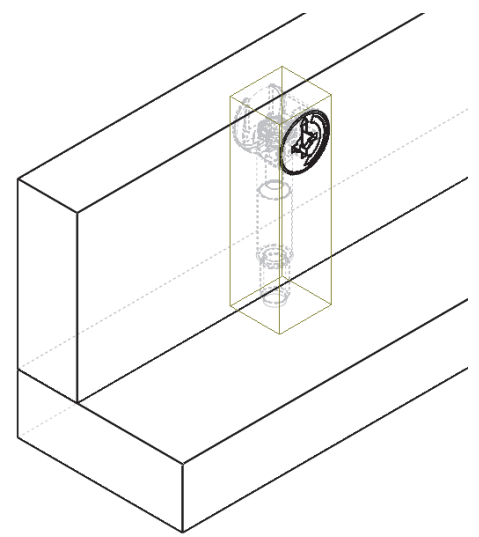

Рис. 4. Эксцентриковая стяжка

Для созданной модели эксцентриковой стяжки была разработана схема автоматизированного размещения основного параметрического элемента, а также связанных с ним монтажных действий, что в общем итоге позволяет в автоматическом режиме изменять размеры в зависимости от размеров соединяемых деталей.

На последнем этапе проектирования изделия предполагается получение конструкторской документации с помощью ранее сделанных шаблонов чертежей, форматов листов и стандартов. При использовании панели свойств вводятся обозначение, наименование, материал и другие важные свойства. Габаритные размеры снимаются автоматически с помощью функции граничной рамки. Пример готового сборочного чертежа представлен на рис. 5.

Аналогичным образом можно получить рабочие чертежи деталей с указанием всех необходимых видов, разрезов и сечений (рис. 6).

Следует отметить, что важнейшее значение имеет соответствие выполняемой конструкторской документации действующим стандартам [17]. Так, в рамках САПР появилась возможность создавать свои собственные редакционные стандарты, эквивалентные установленным стандартам ISO, ANSI, ГОСТ и т. д. Эти стандарты могут позволить сохранить все настройки для одного стандарта, который затем можно передать другим пользователям.

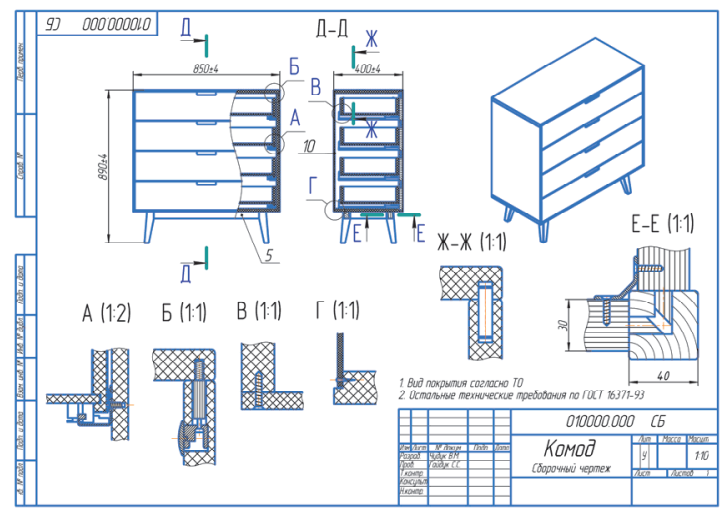

Рис. 5. Сборочный чертеж комода

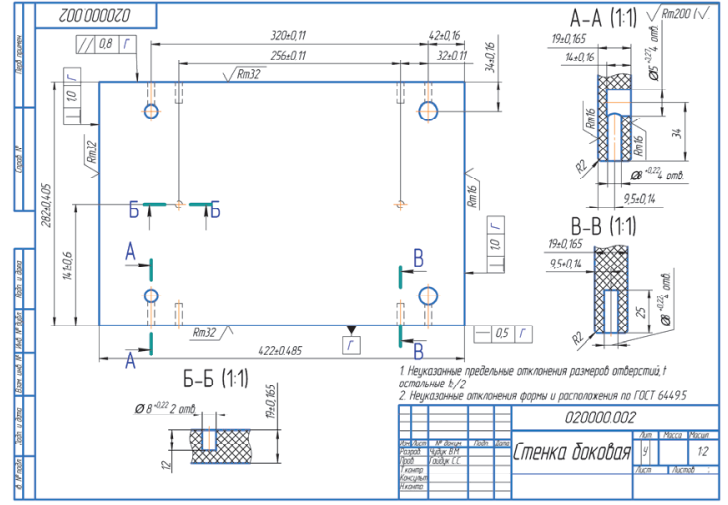

Рис. 6. Рабочий чертеж детали

Заключение. Внедрение систем автоматизированного проектирования в деревообрабатывающую и мебельную промышленность имеет важное значение. Они позволяют значительно сократить время на проектирование (до 20\%) и при этом повысить качество выполняемой конструкторской документации. Наиболее перспективным направлением является внедрение такого подхода, как проектирование в среде многотельной детали. Этот метод позволит на базе простых операций создавать сложные изделия с возможностью разработки полного комплекта конструкторской документации, соответствующей требованиям нормативной документации. Также следует отметить, что предложенная методика позволяет использовать разработанную трехмерную модель изделия для имитации различных условий эксплуатации вместо длительного и дорогостоящего физического моделирования. Предложенная методика проектирования изделий из древесины используется на мебельных предприятиях, что подтверждается актами внедрения. 


\section{Список литературы}

1. Лесной фонд Республики Беларусь. URL: https://mlh.by/our-main-activites/forestry/forests (дата обращения: 01.09.2021).

2. Промышленность Республики Беларусь: стат. сб. / Нац. статист. ком. Респ. Беларусь. Минск: Гос. ком. по имуществу Респ. Беларусь, 2021. 52 с.

3. Стариков А. В., Старикова А. А. Обзор современных САПР для мебельной промышленности // Актуальные направления научных исследований XXI века: теория и практика. 2020. Т. 8, № 1 (48). C. 244-249.

4. Бунаков П. Ю. Автоматизация мебельного предприятия: проблемы и решения // ЛесПромИнформ. 2014. № 2 (100). C. 138-145.

5. Кузнецов С. А. Отечественные САПР для мебельщиков - результат перехода универсальных САПР к специализированным САПР // Промышленные АСУ и контроллеры. 2020. № 5. С. 53-60.

6. Бунаков П. Ю. Современные тенденции развития систем автоматизации проектирования корпусной мебели // Дизайн и производство мебели. 2007. № 1. С. 27-30.

7. Трофимов С. П., Пардаев А. С. Автоматизация конструирования и подготовки производства мебели. Минск: Колорград, 2021. 100 с.

8. Кузнецов С. А. Разработка отечественных специализированных САПР - неизбежный путь к обеспечению технологической независимости России от зарубежных САПР // Приборы и системы. Управление, контроль, диагностика. 2017. № 5. С. 1-11.

9. Бунаков П. Ю. Базис 11: единая среда проектирования // САПР и графика. 2019. № 9 (275). C. 63-67.

10. Багаев Р. Система Базис и ее роль в развитии мебельной компании // САПР и графика. 2019. № 12 (278). C. 76-82.

11. Бужинская Н. В., Закоможный В. И. Особенности разработки 3D-дизайна мебели // Инновационные технологии в науке нового времени: сб. ст. Междунар. науч.-практ. конф., Уфа, 1 февр. 2017 г., Уфа: Аэтерна, 2017. С. 62-64.

12. Бунаков П. Ю., Колесников Р. А. К вопросу о выборе 3D ядра для специализированной САПР корпусной мебели // Актуальные направления научных исследований XXI века: теория и практика. 2015. Т. 3, № 5-4 (16-4). С. 438-442.

13. Демитрова И. П., Назаров А. И. Проектирование мебели в SolidWorks // Актуальные направления научных исследований XXI века: теория и практика. 2015. Т. 3, № 5-4 (16-4). С. 452-455.

14. Bethune J. D. Engineering design and graphics with SolidWorks 2016. Boston: Pearson, 2017. 829 p.

15. Зиновьев Д. Основы проектирования в SolidWorks 2016. Павлоград: Студия Vertex, 2017. 277 с.

16. Lombard M. SolidWorks 2009 Bible. Wiley Publishing, Inc., 2009. 1177 p.

17. Единая система конструкторской документации. Общее положение: ГОСТ 2.001-2013. Введ. 01.09.2016. Минск: Госстандарт, 2016. 8 с.

\section{References}

1. Forest Fund of the Republic of Belarus. Available at: https://mlh.by/our-main-activites/forestry/forests (accessed 01.09.2021) (In Russian).

2. Promyshlennost' Respubliki Belarus': statisticheskiy sbornik [Industry of the Republic of Belarus: statistical compilation]. Minsk, Gosudarstvennyy komitet po imushchestvy Respubliki Belarus' Publ., 2021. 52 p. (In Russian)

3. Starikov A. V., Starikova A. A. An overview of modern CAD systems for the furniture industry. Aktual'nyye napravleniya nauchnykh issledovaniy XXI veka: teoriya i praktika [Actual directions of scientific research in the XXI century: theory and practice], 2020, vol. 8, no. 1 (48), pp. 244-249 (In Russian).

4. Bunakov P. Yu. Furniture factory automation: problems and solutions. LesPromInform [LesPromInform], 2014, no. 2 (100), pp. 138-145 (In Russian).

5. Kuznetsov S. A. Domestic CAD systems for furniture makers - the result of the transition from universal CAD systems to specialized CAD systems. Promyshlennyye ASU i kontrollery [Industrial ACS and controllers], 2020, no. 5, pp. 53-60. (In Russian).

6. Bunakov P. Yu. Modern trends in the development of automation systems for the design of cabinet furniture. Dizayn i proizvodstvo mebeli [Furniture design and production], 2007, no. 1, pp. 27-30 (In Russian).

7. Trofimov S. P., Pardaev A. S. Avtomatizatsiya konstruirovaniya i podgotovki proizvodstva mebeli [Furniture design and preparation automation]. Minsk, Kolorgrad Publ., 2021. 100 p. (In Russian).

8. Kuznetsov S. A. Development of domestic specialized CAD - an inevitable way to ensure the technological independence of Russia from foreign CAD. Pribory i sistemy. Upravleniye, kontrol', diagnostika [Instruments and systems. Management, control, diagnostics], 2017, no. 5, pp. 1-11 (In Russian). 
9. Bunakov P. Yu. Basis 11: a unified design environment. SAPR i grafika [CAD and graphics], 2019, no. 9 (275), pp. 63-67 (In Russian).

10. Bagaev R. Basis system and its role in the development of a furniture company. SAPR i grafika [CAD and graphics], 2019, no. 12 (278), pp. 76-82 (In Russian).

11. Buzhinskaya N. V., Zakomozhny V. I. Features of the development of 3D furniture design. Innovatsionnyye tekhnologii v nauke novogo vremeni: sbornik statey Mezhdunarodnoy nauchno-prakticheskoy konferentsii [Innovative technologies in the science of modern times: collection of articles of the International Scientific and Practical Conference]. Ufa, 2017, pp 62-64 (In Russian).

12. Bunakov P. Yu., Kolesnikov R. A. On the issue of choosing a 3D kernel for specialized CAD of cabinet furniture. Aktual'nyye napravleniya nauchnykh issledovaniy XXI veka: teoriya i praktika [Actual directions of scientific research of the XXI century: theory and practice], 2015, vol. 3, no. 5-4 (16-4), pp. 438-442 (In Russian).

13. Demitrova I. P., Nazarov A. I. Furniture design in SolidWorks. Aktual'nyye napravleniya nauchnykh issledovaniy XXI veka: teoriya i praktika [Actual directions of scientific research of the XXI century: theory and practice], 2015, vol. 3, no. 5-4 (16-4), pp. 452-455 (In Russian).

14. Bethune J. D. Engineering design and graphics with SolidWorks 2016. Boston, Pearson, 2017. 829 p.

15. Zinoviev D. Osnovy proyektirovaniya v SolidWorks 2016 [Basics of design in SolidWorks 2016]. Pavlograd, Studio Vertex Publ., 2017. 277 p. (In Russian).

16. Lombard M. SolidWorks 2009 Bible. Wiley Publishing, Inc., 2009. 1177 p.

17. GOST 2.001-2013. Unified system of design documentation. General provisions. Minsk, Gosstandart Publ., 2016. 8 p. (In Russian).

\section{Информация об авторах}

Гайдук Сергей Сергеевич - кандидат технических наук, доцент кафедры технологии и дизайна изделий из древесины. Белорусский государственный технологический университет $(220006$, г. Минск, ул. Свердлова, 13a, Республика Беларусь). E-mail: haiduk@belstu.by

Чудук Владимир Михайлович - инженер-технолог. ЧПТУП «Линалис плюс» (222744, г. Дзержинск, 1-я Ленинская ул., 43, Республика Беларусь). E-mail: chuduk.vladimir@tut.by

\section{Information about the authors}

Haiduk Siarhei Siargeevich - PhD (Engineering), Assistant Professor, the Department of Technology and Design of Wooden Articles. Belarusian State Technological University (13a, Sverdlova str., 220006, Minsk, Republic of Belarus). E-mail: haiduk@belstu.by

Chuduk Vladimir Mikhaylovich - engineer-technologist. PPTUE "Linalis plus" (43, 1st Leninskaya str., 222744, Dzerzhinsk, Republic of Belarus). E-mail: E-mail: chuduk.vladimir@tut.by 